\title{
INVESTIGATION AND FEASIBILITY OF FLY ASH AND RISE HUSK ASH AND QUARRY SAND IN M-30 CONCRETE
}

\author{
${ }^{1}$ Abhijeet Nardey and ${ }^{2}$ A. R. Gajbhiye \\ ${ }^{1}$ Assistant professor in Civil Engineering Department, RGCER, Nagpur\} \\ ${ }^{2}\{$ Professor in Civil Engineering Department, Yeshwantrao Chavan College of Engineering, Nagpur\}
}

\begin{abstract}
The proposed research is aimed at investigating the performance of rise husk ash and fly ash and quarry dust and black sand as partial replacement of standard sand in concrete. The study also includes investigation of rise husk ash and fly ash in different proportion in different grades of concrete. The research outcome may also be beneficial in developing and utilizing locally available rise husk ash and fly ash in abundant quantity. The studies also include fly ash used as a admixture or pozzolana in concrete making so in current condition no study available on the use of fly ash, rise husk ash with quarry dust as partial replacement of sand in concrete.
\end{abstract}

Key Words : Fly Ash, RHA, Quarry Sand

\section{Introduction}

Incorporating fly ashes of both types (low and high calcium) in cementations systems is nowadays considered a common practice in the construction sector. However, the fly ash quantities that are produced globally are steadily increasing, exceeding the utilization rates that in most countries remain low. In Greece, a recent report (Papadakos V.G. 2010) revealed that although about 10 million tons of fly ash is generated annually, their absorption in several applications (mainly the cement industry) is stuck at $10 \%$. The amounts that remain unused obviously create acute environmental problems and moreover inhibit the path towards sustainability (Mehta K. 1998, Fraay A.L.A. 1989). For increasing the utilization rate of this by-product, it is necessary to fully explore its dynamic and pozzolana potential, but also to come up with methods of enhancing its slow reaction. This task however becomes difficult when dealing with a very heterogeneous product, where not all streams are the same, chemically or physically, and furthermore, when the effect of each of those parameters on the hydration of fly ash/cement (FC) systems has not yet been completely understood. Raman S. N. et al. (2008), reports the experimental study undertaken to investigate the influence of partial replacement of sand with quarry dust, and cement with fly ash on the concrete compressive strength development. Two types of replacement proportion of sand with quarry dust, $20 \%$ and $40 \%$ were practiced in the concrete mixes except in the control concrete mix. Besides, replacement proportion of $10 \%$ cement content with fly ash was practiced in some of the concrete mixes. Two types of curing methods, water curing and air curing under controlled laboratory conditions were practiced during the entire study.
Recorded results indicate that concrete incorporating quarry dust without the inclusion of fly ash exhibited lower compressive strength than the control concrete at all ages. This weakness was overcome by the inclusion of fly ash into the quarry dust concrete in which it resulted in the enhanced compressive strength at almost all conditions. It can be concluded that quarry dust can be utilized as partial replacement material to sand, in the presence of fly ash, to produce concretes with fair ranges of compressive strength. Satakhun Detphan et al. (2008), presents the fundamental data of fly ash based geopolymer mixed with the open field rice husk ash, the basic properties viz., setting time, burning temperature of rice husk heap, temperature and $\mathrm{pH}$ during mixing were presented, furthermore, the 7 days strength of geopolymer mortar replaced by rice husk ash of $0,20,40$ and $60 \%$ were also investigated. Alireza Naji Givi et al. (2010), presents an overview of the work carried out on the use of RHA as partial replacement of cement in mortar and concrete. Habeeb G.A. et al. (2009) \& Muhammad Harunur Rashid et al. (2010) conducted an experimental investigation on the influence of Rice Husk Ash (RHA) Average Particle Size (APS) on the mechanical properties and drying shrinkage of the produced RHA blended concrete. Dao Van Dong etal. (2008) presents several key properties of high a five-space indentation. A colon is inserted before an equation is presented, but there is no punctuation following the equation. All equations are numbered and referred to in the text solely by a number enclosed in a round bracket (i.e., (3) reads as "equation $3 ")$. Ensure that any miscellaneous numbering system you use in your paper cannot be confused with a reference [4] or an equation (3) designation. The first aspect is economics. In most of market fly ash is less expensive than Portland cement. Therefore as replacement level of 
fly ash increases the cost to produce concrete decreases. The second aspect and arguably the most important is the environment. As it is industrial by-product much which is deposited in landfill if not used in concrete. As more fly ash use less demand for Portland cement. So it will lower $\mathrm{CO} 2$ emission. Final aspect influencing the use of High replacement level is technical benefit of high volume of fly ash concrete (HVFAC).HVFAC has improved performance over ordinary Portland cement concrete, especially in term of durability when appropriate used.

\section{Chemical composition of Cement in \% of RHA and FA are near about same.}

\begin{tabular}{|c|c|c|c|c|c|c|c|c|c|}
\hline $\begin{array}{l}\text { Materi } \\
\text { al }\end{array}$ & $\mathrm{Si02}$ & $\begin{array}{l}\text { Al2 } \\
\text { O3 }\end{array}$ & $\begin{array}{l}\mathrm{Fe} 2 \\
03\end{array}$ & $\begin{array}{l}\mathrm{Ca} \\
\mathrm{o}\end{array}$ & $\begin{array}{l}\text { Mg } \\
\text { o }\end{array}$ & $\begin{array}{l}\text { LO } \\
\text { I }\end{array}$ & $\begin{array}{l}\text { SO } \\
3\end{array}$ & $\begin{array}{l}\text { K2 } \\
\mathrm{o}\end{array}$ & $\begin{array}{l}\mathrm{Na} 2 \\
\mathrm{O} 3\end{array}$ \\
\hline cement & $\begin{array}{l}19 . \\
7\end{array}$ & 5.2 & 3.73 & $\begin{array}{l}62 . \\
9\end{array}$ & 2.5 & $\begin{array}{l}0.9 \\
6\end{array}$ & $\begin{array}{l}2.7 \\
2\end{array}$ & $\begin{array}{l}0.9 \\
0\end{array}$ & 0.25 \\
\hline Fly ash & 40 & 25 & 6 & 20 & $\begin{array}{l}3.7 \\
1\end{array}$ & 3.0 & 1.7 & $\begin{array}{l}0.8 \\
0\end{array}$ & 0.96 \\
\hline $\begin{array}{l}\text { Rise } \\
\text { husk } \\
\text { ash }\end{array}$ & $\begin{array}{l}78 . \\
21\end{array}$ & \multicolumn{2}{|c|}{$\begin{array}{l}\mathrm{Si02+} \\
\mathrm{Al} 2 \mathrm{O3}+ \\
\mathrm{Fe} 203=82.64\end{array}$} & $\begin{array}{l}0.9 \\
9\end{array}$ & $\begin{array}{l}4.8 \\
9\end{array}$ & & & & \\
\hline
\end{tabular}

\subsection{Materials}

I. MATERIAL TO BE COLLECTED

I. Fly ash will be collected from koradi khaperkheda power plant.

II. Rise husk ash will be collected from chimur district from ash agro limited.

III. Quarry dust will be collected from Sidheshwar crushing plant panchgao.

\subsection{Material Selection}

1. Cement (OPC 53 Grade)

2. Rice Husk Ash (RHA)

3. Fly Ash (FA)

4. Quarry Dust (Stone Sand)

5. Fine aggregate

6. Coarse aggregate

2.3 Experimental Methodology

$>$ Collection of Materials

$>$ Weight Batching

$>\quad$ Test on Concrete

$>$ Casting of Concrete Cube, cylinder, Beam

$>$ Compaction

By Hand

By Vibrator

$>$ Curing

$>$ Specimen Testing

$>$ Compression Testing

$>$ Test Result

\subsection{Test that will perform}

$>$ Compressive strength on cube (150mmx150mmx150mm)

$>\quad$ Flexural strength on beam (150mmx150mmx750mm)

$>$ Split tensile test on cylinder as per IS-516 (150mm diameter and $300 \mathrm{~mm}$ height)

$>$ Workability slump test, vee bee test.

\section{Figures and Tables}

3.1 Compressive strength of concerte.

\begin{tabular}{|c|c|c|c|c|c|}
\hline & & \multicolumn{4}{|c|}{ Compressive Strength } \\
\hline Mix Type & $\begin{array}{c}\text { Identificat } \\
\text { ion } \\
\text { Symbol } \\
\end{array}$ & $\begin{array}{l}7 \mathrm{da} \\
\text { ys }\end{array}$ & $\begin{array}{c}14 \\
\text { day } \\
\text { s }\end{array}$ & $\begin{array}{c}28 \\
\text { day } \\
\mathrm{s} \\
\end{array}$ & $\begin{array}{c}90 \\
\text { day } \\
\text { s }\end{array}$ \\
\hline \multicolumn{6}{|l|}{$\begin{array}{c}\text { Phase 1-- } \\
\text { Combination } \\
\text { FA+RHA } \\
\end{array}$} \\
\hline Controlled Mix & M30-A & 23.5 & 28.7 & 36.5 & 42.2 \\
\hline CM70\%+FA30\% & M30-A1 & 15.5 & 23.6 & $\begin{array}{c}31.2 \\
5 \\
\end{array}$ & $\begin{array}{c}33.2 \\
4 \\
\end{array}$ \\
\hline $\begin{array}{c}\text { CM } 70 \%+\mathrm{FA} \\
27.5 \%+2.5 \% \text { RHA }\end{array}$ & M30-A2 & 20.5 & 24 & 32.5 & $\begin{array}{c}34.8 \\
5 \\
\end{array}$ \\
\hline $\begin{array}{c}\text { CM } 70 \%+\text { FA } 25 \% \\
+5 \% \text { RHA } \\
\end{array}$ & M30-A3 & $\begin{array}{c}23.8 \\
9 \\
\end{array}$ & 31.1 & 32.2 & $\begin{array}{c}35.5 \\
5 \\
\end{array}$ \\
\hline $\begin{array}{c}\text { CM } 70 \%+F A \\
22.5 \%+7.5 \% \text { Rha }\end{array}$ & M30-A4 & 24.1 & $\begin{array}{c}32.7 \\
8 \\
\end{array}$ & 37.1 & $\begin{array}{c}38.1 \\
5 \\
\end{array}$ \\
\hline $\begin{array}{c}\text { CM } 70 \%+\text { FA } 20 \%+10 \\
\% \text { RHA }\end{array}$ & M30-A5 & 23.6 & 32.5 & 35.1 & 36.8 \\
\hline \multicolumn{6}{|l|}{$\begin{array}{c}\text { Phase2- } \\
\text { combination } \\
\text { FA+RHA+QS } \\
\end{array}$} \\
\hline Controlled Mix & M30-A & 23.5 & 28.7 & 36.5 & 42.2 \\
\hline $\begin{array}{c}\mathrm{CM} 70 \%+\mathrm{FA} 30 \%+15 \\
\% \mathrm{QS} \\
\end{array}$ & M30-B1 & 26.5 & $\begin{array}{c}36.4 \\
4 \\
\end{array}$ & $\begin{array}{c}37.3 \\
2 \\
\end{array}$ & 38.9 \\
\hline $\begin{array}{c}\mathrm{CM} 70 \%+\mathrm{FA} \\
25 \%+5 \% \mathrm{RHA}+15 \% \mathrm{Q} \\
\mathrm{S} \\
\end{array}$ & M30-B2 & $\begin{array}{c}28.1 \\
5\end{array}$ & $\begin{array}{c}37.6 \\
2\end{array}$ & $\begin{array}{c}39.1 \\
5\end{array}$ & 40.3 \\
\hline $\begin{array}{c}\text { CM } 70 \%+\mathrm{FA} \\
22.5 \%+7.5 \% \text { RHA }+15 \\
\% \mathrm{QS} \\
\end{array}$ & M30-B3 & $\begin{array}{c}30.2 \\
2\end{array}$ & 38 & 40.5 & $\begin{array}{c}42.3 \\
5\end{array}$ \\
\hline $\begin{array}{c}\mathrm{CM} 70 \%+\mathrm{FA} 30 \%+30 \\
\% \mathrm{QS} \\
\end{array}$ & M30-B4 & 28 & $\begin{array}{c}38.4 \\
5 \\
\end{array}$ & 40.8 & 44 \\
\hline $\begin{array}{c}\text { CM } 70 \%+F A \\
25 \%+5 \% \text { RHA }+30 \% \mathrm{Q} \\
\mathrm{S} \\
\end{array}$ & M30-B5 & 30.4 & 39.8 & 41.6 & 42.8 \\
\hline $\begin{array}{c}\mathrm{CM} 70 \%+\mathrm{FA} \\
22.5 \%+7.5 \% \mathrm{RHA}+30 \\
\% \mathrm{QS} \\
\end{array}$ & M30-B6 & 31.2 & 32.5 & 43 & 45.4 \\
\hline $\begin{array}{c}\mathrm{CM} 70 \%+\mathrm{FA} 30 \%+45 \\
\% \mathrm{QS} \\
\end{array}$ & M30-B7 & $\begin{array}{c}29.7 \\
7 \\
\end{array}$ & 36.2 & 38.2 & 40.7 \\
\hline $\begin{array}{c}\mathrm{CM} 70 \%+\mathrm{FA} \\
25 \%+5 \% \mathrm{RHA}+45 \% \mathrm{Q} \\
\mathrm{S} \\
\end{array}$ & M30-B8 & 31.4 & 37 & 37.5 & 42.5 \\
\hline $\begin{array}{c}\text { CM } 70 \%+\mathrm{FA} \\
22.5 \%+7.5 \% \mathrm{RHA}+45 \\
\% \mathrm{QS} \\
\end{array}$ & M30-B9 & 31 & 38.2 & $\begin{array}{c}40.2 \\
2\end{array}$ & 43.8 \\
\hline
\end{tabular}



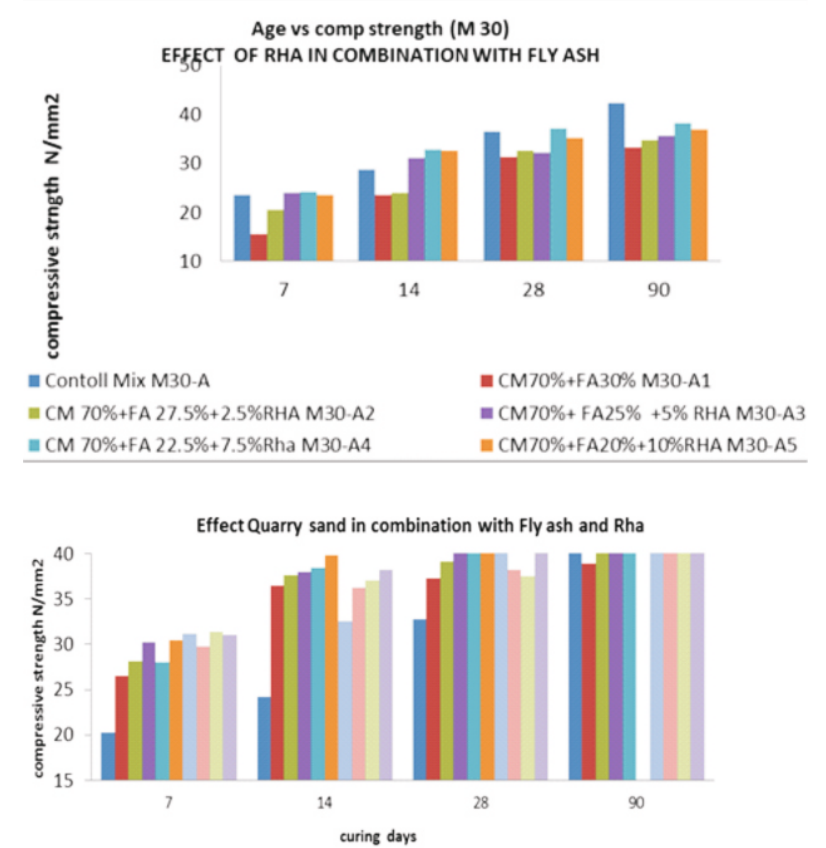

- Contoll Mix M3O-A

- CM70\%\$FA30\% +15\%OS M3O-B1

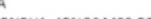

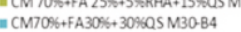

IICM 70\%+FA 22.5\%+7.5\%6RHA+30\% 05 M30-86

- CM 70\%+FA 22.5\%+7.5\%6RA +15\%OS M30-B3 $=C M$ 70\%+FA 25\% +5\%RHA+30\%6OS M $30-85$

$=$ CM 70\%+FA 22.5\%+7.5\% RHAA+45\%OS M $30-89$

Flexural Strength

\begin{tabular}{|c|c|c|c|}
\hline \multirow{2}{*}{$\begin{array}{c}\text { Phase-1(Replacement } \\
\text { cement by FA and RHA) } \\
\text { Mix-M-30 }\end{array}$} & \multirow[b]{2}{*}{ Identification } & \multicolumn{2}{|c|}{$\begin{array}{c}\text { Flexural strength } \\
\mathrm{N} / \mathrm{mm} 2\end{array}$} \\
\hline & & 28 days & 90 days \\
\hline Control Mix & M30-A & 4.14 & 4.45 \\
\hline $\mathrm{CM} 70 \%+\mathrm{FA} 30 \%$ & M30-A1 & 3.19 & 3.57 \\
\hline $\begin{array}{l}\text { CM } 70 \%+\text { FA } 27.5 \%+\text { RHA } \\
2.5 \%\end{array}$ & M30-A2 & 3.35 & 3.85 \\
\hline CM $70 \%+$ FA $25 \%+$ RHA $5 \%$ & M30-A3 & 3.85 & 3.95 \\
\hline $\begin{array}{l}\text { CM70\%+FA22.5\%+RHA } \\
7.5 \%\end{array}$ & M30-A4 & 4.15 & 4.35 \\
\hline CM70\%+FA20\%+RHA $10 \%$ & M30-A5 & 3.86 & 3.52 \\
\hline $\begin{array}{l}\text { Phase }-2 \text { (Replacement } \\
\text { natural sand by quarry sand } \\
\text { and cement by FA and } \\
\text { RHA) }\end{array}$ & & & \\
\hline Control Mix & M30-A & 4.14 & 4.45 \\
\hline CM70\%+FA30\%+15\%QS & M30-B1 & 3.41 & 3.81 \\
\hline $\begin{array}{l}\mathrm{CM} 70 \%+\mathrm{FA} \\
25 \%+5 \% \text { RHA }+15 \% \mathrm{QS} \\
\end{array}$ & M30-B2 & 3.53 & 3.75 \\
\hline $\begin{array}{l}\text { CM 70\%+FA } \\
22.5 \%+7.5 \% \text { RHA }+15 \% \mathrm{QS}\end{array}$ & M30-B3 & 3.68 & 3.85 \\
\hline CM70 $\%+\mathrm{FA} 30 \%+30 \% \mathrm{QS}$ & M30-B4 & 3.75 & 3.87 \\
\hline $\begin{array}{l}\text { CM } 70 \%+\mathrm{FA} \\
25 \%+5 \% \mathrm{RHA}+30 \% \mathrm{QS}\end{array}$ & M30-B5 & 3.88 & 3.95 \\
\hline $\begin{array}{l}\text { CM 70\%+FA } \\
22.5 \%+7.5 \% \text { RHA }+30 \% \mathrm{QS} \\
\end{array}$ & M30-B6 & 4.35 & 4.45 \\
\hline & & 3.66 & 3.81 \\
\hline CM70\%+FA30 $\%+45 \% \mathrm{QS}$ & M30-B7 & 3.68 & 3.72 \\
\hline $\begin{array}{l}\text { CM } 70 \%+\mathrm{FA} \\
25 \%+5 \% \mathrm{RHA}+45 \% \mathrm{QS}\end{array}$ & M30-B8 & 3.75 & 3.85 \\
\hline $\begin{array}{l}\text { CM } 70 \%+\mathrm{FA} \\
22.5 \%+7.5 \% \text { RHA }+45 \% \mathrm{QS}\end{array}$ & M30-B9 & 4.14 & 4.45 \\
\hline
\end{tabular}

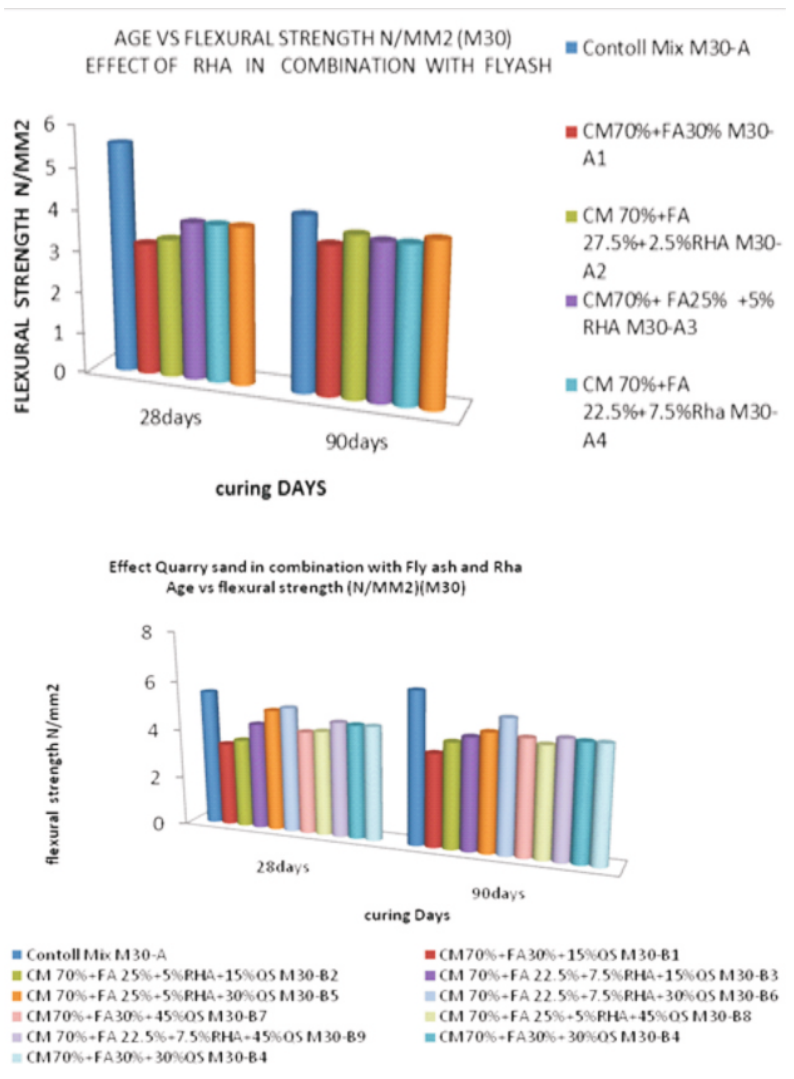

Split tensile Strength

EFFECT OF RHA IN COMBINATION WITH FLYASH Age Vs split tensile strength $(\mathrm{N} / \mathrm{mm} 2)(\mathrm{M} 30)=$ Contoll Mix M $30-A$
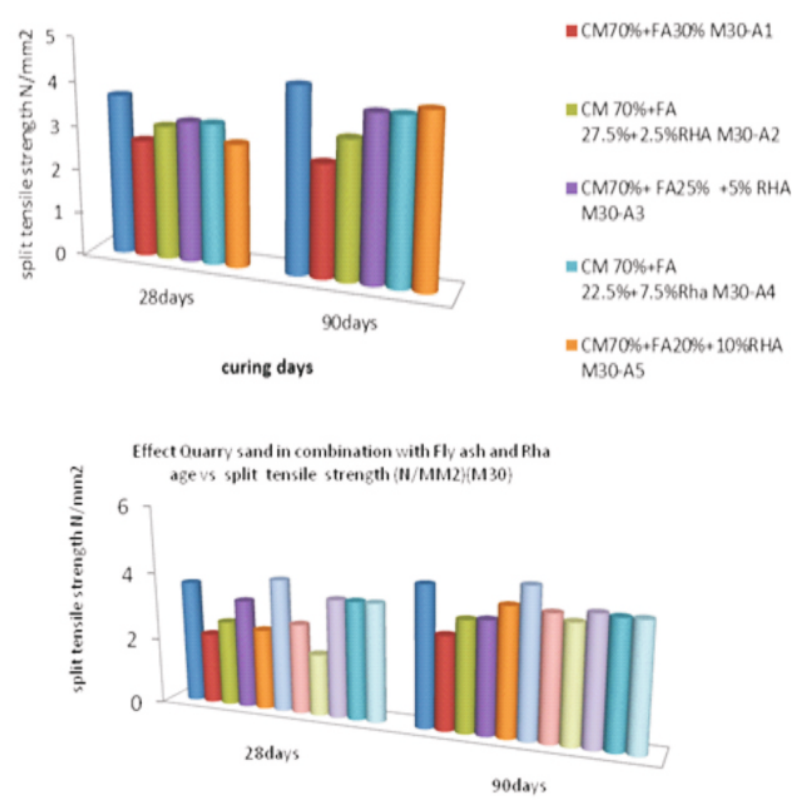

curing Days

- Contollmix M30-A

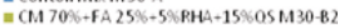

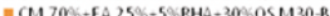

$=C M 700+F A 300+45 \div 05 M 30 B 7$

- $C M 70 \%+F A 30 \%+15 \div 05 M 30 B 1$

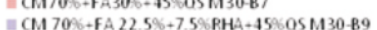

= C1.70\%-FA22.5\%-7 S\%PHA-150\%OSH30-83 CM 705-FA 22.5\%+7.5 $\triangle \mathrm{PHA}+30 \leq 05 \mathrm{M} 30-\mathrm{B} 6$ CM $700-F A 25 \%+5 \%$ PHA $45 \% 051430-88$

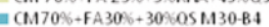




\begin{tabular}{|c|c|c|c|}
\hline $\begin{array}{l}\text { Phase-1(Replacement cement by FA } \\
\text { and RHA) }\end{array}$ & & \multicolumn{2}{|c|}{$\begin{array}{l}\text { Split tensile } \\
\text { strength } \\
\mathrm{N} / \mathrm{mm} 2\end{array}$} \\
\hline Mix-M-30 & Identification & $\begin{array}{l}28 \\
\text { days }\end{array}$ & $\begin{array}{l}90 \\
\text { days }\end{array}$ \\
\hline Control Mix & M30-A & 3.67 & 4.2 \\
\hline $\mathrm{CM} 70 \%+\mathrm{FA} 30 \%$ & M30-A1 & 2.69 & 3.57 \\
\hline CM $70 \%+$ FA $27.5 \%+$ RHA $2.5 \%$ & M30-A2 & 3.05 & 3.85 \\
\hline CM $70 \%+$ FA $25 \%+$ RHA $5 \%$ & M30-A3 & 3.2 & 3.75 \\
\hline CM70\%+FA22.5\%+RHA $7.5 \%$ & M30-A4 & 3.26 & 4.15 \\
\hline CM $70 \%+$ FA $20 \%+$ RHA $10 \%$ & M30-A5 & 2.8 & 3.9 \\
\hline $\begin{array}{l}\text { Phase }-2 \text { (Replacement natural sand } \\
\text { by quarry sand and cement by FA } \\
\text { and RHA) }\end{array}$ & & & \\
\hline Control Mix & M30-A & 3.67 & 4.2 \\
\hline $\mathrm{CM} 70 \%+\mathrm{FA} 30 \%+15 \% \mathrm{QS}$ & M30-B1 & 2.12 & 2.8 \\
\hline $\mathrm{CM} 70 \%+\mathrm{FA} 25 \%+5 \%$ RHA $+15 \% \mathrm{QS}$ & M30-B2 & 2.55 & 3.3 \\
\hline $\begin{array}{l}\text { CM } 70 \%+\text { FA } \\
22.5 \%+7.5 \% \text { RHA }+15 \% \mathrm{QS}\end{array}$ & M30-B3 & 3.25 & 3.35 \\
\hline $\mathrm{CM} 70 \%+\mathrm{FA} 30 \%+30 \% \mathrm{QS}$ & M30-B4 & 3.53 & 3.74 \\
\hline $\mathrm{CM} 70 \%+\mathrm{FA} 25 \%+5 \%$ RHA $+30 \% \mathrm{QS}$ & M30-B5 & 2.41 & 3.8 \\
\hline $\begin{array}{ll}\mathrm{CM} & 70 \%+\mathrm{FA} \\
22.5 \%+7.5 \% \mathrm{RHA}+30 \% \mathrm{QS} & \\
\end{array}$ & M30-B6 & 4.31 & 5.89 \\
\hline & & 3.61 & 3.79 \\
\hline $\mathrm{CM} 70 \%+\mathrm{FA} 30 \%+45 \% \mathrm{QS}$ & M30-B7 & 3.67 & 3.79 \\
\hline $\mathrm{CM} 70 \%+\mathrm{FA} 25 \%+5 \%$ RHA $+45 \% \mathrm{QS}$ & M30-B8 & 3.93 & 3.95 \\
\hline $\begin{array}{ll}\mathrm{CM} & 70 \%+\mathrm{FA} \\
22.5 \%+7.5 \% \mathrm{RHA}+45 \% \mathrm{QS} & \\
\end{array}$ & M30-B9 & 3.67 & 4.2 \\
\hline
\end{tabular}

\section{Conclusions}

Compressive strength increases with the increase in the percentage of Fly ash, Rice Husk Ash up to replacement (22.5\%FA and $7.5 \%$ RHA) of Cement without quarry sand. Compressive strength increase by addition of quarry sand in addition to fly ash and rice husk ash. For a mix by replacement of $22.55 \%$ FA and $7.5 \%$ RHA to cement and $30 \%$ quarry sand as a replacement of fine aggregate (sand) gives maximum strength. The Maximum Splitting Tensile is given by Concrete mix of Partial replacement in cement by $22.5 \%$ Fly Ash, $7.5 \%$ RHA and Fine Aggregate by 30\% Quarry Sand. The unit weight of concrete increases uniformly with the increase in Quarry Sand and decreases with the increase of Rice Husk Ash and Fly Ash content.

\section{References}

[1] D. S. Rajendra Prasad, S. M. Maheshwarappa, S. Suresh(3), in this study focuses on utilization of waste Pozzuolana products such as fly ash and Rice Husk Ash (RHA) as an alternative to OPC to produce ternary blended cement with an objective to increase the optimum percentage of replacement of pozzuolana to OPC without affecting the concrete properties. $\mathrm{CO} 2$ curing is carried out to reduce the curing duration without affecting the compressive strength and trying to achieving the 28 days compressive strength in a short period of 18 hours. The study of $\mathrm{CO} 2$ cured specimens kept in air and water for 3 days, 7 days is also carried out along with comparison of the compressive strength of normal concrete.

[2] Nagabhushana and H. Sharada bai(4), in this study of properties of mortar and concrete in which Crushed Rock Powder (CRP) is used as a partial and full replacement for natural sand. For mortar, CRP is replaced at $20 \% 40 \%, 60 \%, 80 \%$ and $100 \%$. The basic strength properties of concrete were investigated by replacing natural sand by CRP at replacement levels of $20 \%, 30 \%$ and $40 \%$. This study reveals that in case of cement mortars, the natural sand can be replaced by Crushed Rock Powder (CRP). The strength of mortar containing $40 \%$ CRP is much higher than normal mortar containing only sand as fine aggregate.

[3] Rinki khot,R.S Deotale,A.R Nardey Department of civil engineering Yeshwantrao Chavan college of engineering ,Nagpur"To Study The Partial Replacement OfCement By FA \& RHAAnd Natural Sand By Quarry Sand In Concrete"

[4] R. S. Deotale, S. H. Sathawane, A.R. NardeDepartment of Civil Engineering, Yeshwantrao Chavan College of Engineering, Nagpur, MH, India 441110“Effect of Partial Replacement of Cementby Fly Ash, Rice Husk Ash with Using Steel Fibres in Concrete"

[5] Satish D. Kene1, Pravin V. Domke2, Sandesh D. Deshmukh3, R.S.DeotaleDepartment of Civil Engineering, Yeshwantrao Chavan College of Engineering, Nagpur, M H, India 441110“Assessment OF Concrete Strength Using FlyAsh and Rice Husk Ash"

[6] S 10262 -2009“IS Method of MixDesign”, Bureau of IndianStandards, New Delhi

[7] IS 516 -1959 "Methods of Tests forstrength of concrete", Bureau ofIndian Standards, New Delhi

[8] IS 456 -2000 "Code of Practice forPlain and Reinforced Concrete",Bureau of Indian Standards, NewDelhi

[9] G. A. Habeeb, M. M. Fayyadh Department of Civil Engineering, Faculty of Engineering, University of Malaya, Malaysia developed "Rice Husk Ash Concrete: the Effect of RHA Average Particle Size on Mechanical Properties and Drying Shrinkage". 